\title{
REVIEW
}

\section{Ocriplasmin for Treatment of Vitreomacular Traction: An Update}

\author{
Mohammed Ali Khan · Julia A. Haller
}

Received: July 22, 2016 / Published online: September 12, 2016

(c) The Author(s) 2016. This article is published with open access at Springerlink.com

\begin{abstract}
Pharmacologic vitreolysis with ocriplasmin, a 27 kilodalton serine protease, is an effective nonsurgical treatment option for vitreomacular traction (VMT). Data from phase III clinical studies, including the Microplasmin for Intravitreal Injection-Traction Release without Surgical Treatment (MIVI-TRUST) and Ocriplasmin for Treatment for Symptomatic Vitreomacular Adhesion Including Macular Hole (OASIS) studies, have demonstrated the treatment efficacy of ocriplasmin for VMT and full-thickness macular hole (FTMH). Subgroup analysis of these clinical

diameter $\leq 1500 \mu \mathrm{m}$, absence of epiretinal membrane, phakic status, and age younger than 65. As a first-in-class therapeutic, ocriplasmin and its side effects have been carefully monitored by the vitreoretinal community. The following categories of related or possibly related adverse events have been identified: acute reduction in visual acuity, ERG changes, dyschromatopsia, retinal tear or detachment, lens subluxation or phacodonesis, abnormal pupillary reflex, retinal vascular changes, and OCT ellipsoid zone alterations. Adverse events have almost all been transient with restoration of visual acuity; however, in select patients, alterations may persist.
\end{abstract} trials as well as post-marketing clinical series have aided in patient selection by identifying features associated with successful pharmacologic release of VMT with ocriplasmin, including adhesion

Enhanced content To view enhanced content for this article go to http://www.medengine.com/Redeem/ F295F0601C8A111C.

M. A. Khan

Retina Division, Department of Ophthalmology,

Doheny Eye Center UCLA, Pasadena, CA, USA

M. A. Khan · J. A. Haller $(\bowtie)$

Retina Service, Wills Eye Hospital, Philadelphia, PA, USA

e-mail: jhaller@willseye.org
Keywords: Macular hole; Ocriplasmin; Pharmacologic vitreolysis; Vitreomacular adhesion; Vitreomacular traction; Vitreoretinal interface

\section{INTRODUCTION}

Degeneration and liquefaction of the vitreous gel with age ultimately leads to formation of a posterior vitreous detachment (PVD), characterized by the separation of the 
posterior cortical vitreous from the internal limiting membrane [1]. While PVD represents a normal process of the aging eye, incomplete separation or focal adhesions of the vitreous gel to the macula may result in abnormalities of the vitreoretinal interface.

Advances in ophthalmic imaging have focused our understanding of disorders involving the vitreomacular interface. In 2013, the International Vitreomacular Traction Study (IVTS) Group introduced an optical coherence tomography (OCT) based system for the classification of vitreomacular interface disease [2]. Specifically, the terms vitreomacular adhesion (VMA), vitreomacular traction (VMT), and full-thickness macular hole (FTMH) were defined. FTMH was defined as a foveal lesion involving all retinal layers. VMA was defined as macular attachment of the vitreous cortex within a $3-\mathrm{mm}$ radius of the fovea without change in retinal morphology. VMT was differentiated from VMA by the presence of retinal morphologic changes, but without FTMH (Fig. 1). Both VMA and VMT could be further classified by the size of adhesion [focal $(\leq 1500 \mu \mathrm{m})$ or diffuse $(>1500 \mu \mathrm{m})]$ as well as the presence or absence of concurrent macular disease. FTMH was classified as primary (due to VMT) or secondary and further defined by size as small $(\leq 250 \mu \mathrm{m})$, medium ( $>250$ and $\leq 400 \mu \mathrm{m})$, or large $(>400 \mu \mathrm{m})$.

Resulting abnormalities in retinal morphology from VMT commonly lead to visual decline, including metamorphopsia, visual field defect, and decreased visual acuity [3]. Surgical management with pars plana vitrectomy (PPV) has long been the mainstay of VMT and FTMH treatment [4, 5]. However, following the U.S. Food and Drug Administration approval of intravitreal

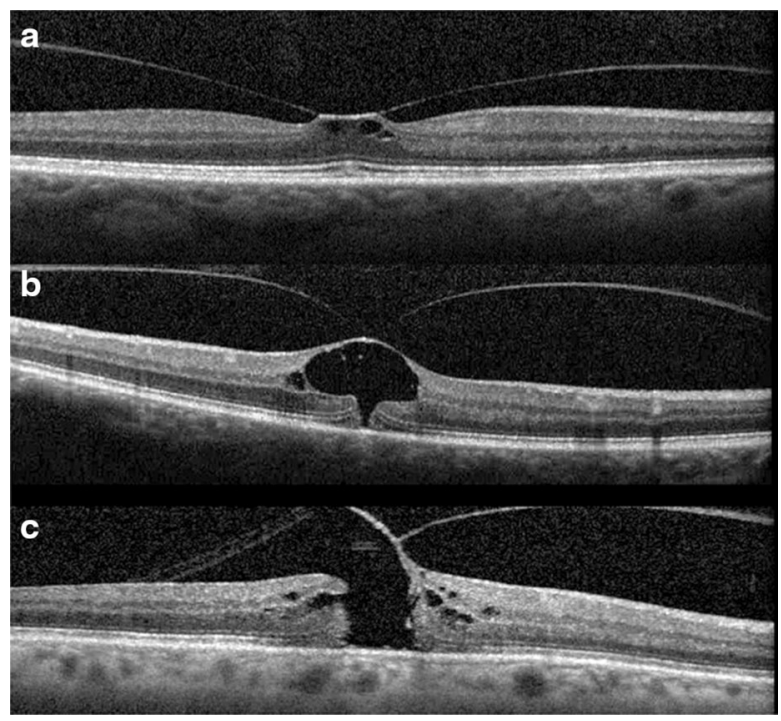

Fig. 1 Examples of vitreomacular traction (VMT) and full thickness macular hole (FTMH). The International Vitreomacular Traction Study (IVTS) Group defined abnormalities of the vitreoretinal interface. Vitreomacular adhesion (VMA) was defined as macular attachment of the vitreous cortex within a $3-\mathrm{mm}$ radius of the fovea without change in retinal morphology. VMT was differentiated from VMA by the presence of retinal morphologic changes (a, b) but without full-thickness defect. FTMH was defined as a foveal lesion involving all retinal layers (c)

ocriplasmin (Jetrea $^{\circledR}$; ThromboGenics, Inc., Iselin, NJ, USA) for the treatment of VMT, pharmacologic vitreolysis is now a viable therapeutic option. Ocriplasmin, a 27 kilodalton serine protease, achieves its effect via lysis of laminin and fibronectin at the vitreoretinal interface and subsequent VMA release [6].

As a first-in-class drug, clinical understanding of ocriplasmin continues to evolve over time. Experience from phase III clinical trials and subsequent post-marketing clinical series have provided added information regarding the efficacy of ocriplasmin, expected "real-world" outcomes, patient selection, and adverse event profiles. These topics are reviewed below. 


\section{Compliance with Ethics Guidelines}

This article is based on previously conducted studies, and does not involve any new studies of human or animal subjects performed by any of the authors.

\section{EFFICACY OF OCRIPLASMIN: CLINICAL TRIAL EVIDENCE}

Data from phase III clinical studies, including the Microplasmin for Intravitreal Injection-Traction Release without Surgical Treatment (MIVI-TRUST) and Ocriplasmin for Treatment for Symptomatic Vitreomacular Adhesion Including Macular Hole (OASIS) studies, have demonstrated the treatment efficacy of ocriplasmin for VMT and FTMH (Table 1).

\section{The Microplasmin for Intravitreal Injection-Traction Release Without Surgical Treatment (MIVI-TRUST) Studies}

Phase III data is available from two multicenter, randomized, double-blind, placebo-controlled clinical trials, referred to collectively as the MIVI-TRUST studies, completed between 2008 and 2010 [6]. Outcomes revealed statistically significant non-surgical achievement of primary and secondary study endpoints as well as improvement in visual acuity and visual function compared to placebo. Based on these results, the U.S. Food and Drug Administration approved use of intravitreal ocriplasmin for the treatment of VMT in 2012.

In the MIVI-TRUST studies, 464 of the 652 enrolled eyes received a single intravitreal injection of $0.125 \mathrm{mg}$ ocriplasmin with follow-up to 6 months. At the day 28 post injection time-point, eyes receiving ocriplasmin exhibited greater release of VMA (primary endpoint, $26.5 \%$ vs. $10.1 \%, p<0.001$ ), closure of macular hole $(40.6 \%$ vs. $10.6 \%$, $p<0.001)$, and presence of full posterior vitreous detachment $(13.4 \%$ vs. $3.7 \%$, $p<0.001)$ compared to eyes receiving vehicle injection [6].

Visual function, as measured by the National Eye Institute Visual Function Questionnaire (NEI VFQ-25), improved in eyes treated with ocriplasmin in the MIVI-TRUST trials. Varma

Table 1 Data from phase III clinical trials: the Microplasmin for Intravitreal Injection-Traction Release without Surgical Treatment

(MIVI-TRUST) and Ocriplasmin for Treatment for Symptomatic Vitreomacular Adhesion Including Macular Hole (OASIS) studies

\begin{tabular}{|c|c|c|c|c|c|c|c|c|c|}
\hline Study & Design & $\begin{array}{l}\text { Patient } \\
\text { number, } \\
\text { ocriplasmin }\end{array}$ & $\begin{array}{l}\text { Patient } \\
\text { number, } \\
\text { vehicle } \\
\text { control }\end{array}$ & $\begin{array}{l}\text { VMT } \\
\text { release } \\
\text { rate } \\
(\%)\end{array}$ & $\begin{array}{l}p \text { value } \\
\text { versus } \\
\text { control }\end{array}$ & $\begin{array}{l}\text { FTMH } \\
\text { closure } \\
\text { rate }(\%)\end{array}$ & $\begin{array}{l}p \text { value } \\
\text { versus } \\
\text { control }\end{array}$ & $\begin{array}{l}\text { Follow-up } \\
\text { interval } \\
\text { (months) }\end{array}$ & $\begin{array}{l}\text { ERG } \\
\text { sub-study? }\end{array}$ \\
\hline $\begin{array}{l}\text { MIVI-TRUST } \\
\text { studies } \\
\text { (aggregate) }\end{array}$ & $\begin{array}{l}\text { RCT, } \\
\text { Phase } \\
\text { III }\end{array}$ & 464 & 188 & 26.5 & $<0.001$ & 40.6 & $<0.001$ & 6 & No \\
\hline OASIS & $\begin{array}{l}\text { RCT, } \\
\text { Phase } \\
\text { III }\end{array}$ & 146 & 74 & 41.7 & $<0.001$ & 30 & 0.163 & 24 & Yes \\
\hline
\end{tabular}

$V M T$ vitreomacular traction, FTMH full-thickness macular hole, $R C T$ randomized controlled trial, ER $G$ electroretinogram, MIVI-TRUST Microplasmin for Intravitreal Injection-Traction Release without Surgical Treatment, OASIS Ocriplasmin for Treatment for Symptomatic Vitreomacular Adhesion Including Macular Hole 
et al. revealed that eyes treated with ocriplasmin had greater mean improvement in baseline NEI VFQ-25 scores (mean change +3.4 versus 0.7 , $p=0.005)$ and were more likely to have a $>5$-point improvement in VFQ-25 scores $36.0 \%$ versus $27.2 \%, p=0.03$ ) compared to eyes treated with vehicle injection [7]. In addition, eyes treated with ocriplasmin had greater improvement in multiple VFQ-25 sub-scale scores, including general vision $(p=0.003)$, distance vision activities $(p=0.03)$, and driving difficulty $(p=0.03)$, and were less likely to have $>5$-point worsening of VFQ-25 composite scores $(15.0 \%$ vs. $24.3 \%, p=0.005)$ compared to the placebo group [7].

Gandorfer et al. similarly analyzed visual function in eyes enrolled in the MIVI-TRUST trials, with added insight as to best-corrected visual acuity (BCVA) [8]. The authors noted that $\geq 2$-line improvement in BCVA was more likely in eyes treated with ocriplasmin at month 6 compared to vehicle injection $(28.0 \%$ vs. $17.1 \%$, $p=0.003)$. Moreover, achievement of VMA release or nonsurgical FTMH closure was strongly associated with visual acuity gains. Multivariate analysis revealed that in eyes with VMT treated with ocriplasmin, resolution of VMA at day 28 was associated with $\geq 2$-line improvement in BCVA $[p=0.006$, odds ratio (OR) 2.023]. This was also true in eyes with FTMH, as nonsurgical hole closure at day 28 was associated with $\geq 2$-line improvement in BCVA $(p<0.001$, OR 6.716).

Haller et al. performed post hoc subgroup analysis of the MIVI-TRUST data to better identify features associated with VMA release with ocriplasmin treatment [9]. The authors reported age younger than 65, VMA adhesion diameter $\leq 1500 \mu \mathrm{m}$, phakic status, presence of FTMH, and absence of ERM as factors associated with nonsurgical resolution of VMA at day 28. In regards to FTMH, non-surgical hole closure at month 6 was correlated strongly with hole size, as closure was achieved in $58.3 \%$ of holes $\leq 250 \mu \mathrm{m}$ ( $p<0.001$ versus vehicle), $36.8 \%$ of holes $>250$ and $\leq 400 \mu \mathrm{m}(p=0.009$ versus vehicle), and in $0 \%$ of holes $>400 \mu \mathrm{m}$.

\section{Ocriplasmin for Treatment for Symptomatic Vitreomacular Adhesion Including Macular Hole (OASIS)}

Additional clinical trial data is available from the OASIS study [10]. In this trial, 220 eyes were randomized in a 2:1 fashion to either a single injection of $0.125 \mathrm{mg}$ ocriplasmin (146 eyes) or vehicle injection (74 eyes). Follow-up in this study was 24 months, considerably longer than that of the MIVI-TRUST trials. In this study, statistically significant nonsurgical release of VMT was observed in eyes treated with ocriplasmin compared to vehicle injection $(41.7 \%$ versus $6.2 \%, p<0.01$ ), similar to the findings of the MIVI-TRUST trials. While FTMH closure occurred at a higher rate in the ocriplasmin group, this did not reach statistical significance $(30 \%$ versus $15.4 \%, p=0.163$ ) [10].

OASIS data, with longer follow-up, also revealed that the statistically significant difference in VMT release rate with ocriplasmin was maintained to 24 months post-treatment. In addition, eyes initially treated with ocriplasmin were more likely to achieve $\mathrm{a} \geq 2$-line gain in visual acuity at 2 years $(50.5 \%$ versus $39.1 \%, p=0.114)$, regardless of initial VMT release and need for subsequent vitrectomy, though this did not achieve statistical significance.

\section{'REAL-WORLD' CLINICAL OUTCOMES AND PATIENT SELECTION}

Following commercial availability of ocriplasmin, several clinical series have been published which offer "real world" clinical 
outcomes with pharmacologic vitreolysis and provide additional insights regarding patient selection. Overall, factors associated with VMT release from "real-world" clinical outcomes were similar to those reported in post hoc analysis of the MIVI-TRUST data (Table 2). Although helpful in describing outcomes and offering criteria to maximize VMT release rates, these series are small, may include patients with co-morbid macular diseases, and may reflect a post hoc selection bias.

\section{Outcomes and Factors Associated with VMT Release}

Singh et al. [11] and Kim et al. [12] retrospectively reported outcomes in series of 17 and 19 eyes, respectively, treated with intravitreal ocriplasmin. VMT release rates were found to be $47.1 \%$ and $42.1 \%$, respectively, and FTMH closure rates of $80 \%$ and $50 \%$, respectively. Rates of release were found commensurate or better than the MIVI-TRUST data. Furthermore, Singh et al. noted that VMT release rate improved if three of the following four criteria were met: adhesion diameter $\leq 1500 \mu \mathrm{m}$, absence of ERM, phakic status, and age younger than 65. If all four criteria were met, the release rate further improved to $75 \%$ (3/4 eyes). Kim et al. also reported that adhesion diameter $\leq 1500 \mu \mathrm{m}$, absence of ERM, phakic status, and age younger than 65 were associated with VMT release in their study.

More recently, larger series were published by Warrow et al. [13] and Sharma et al. [14]. Warrow et al. reported outcomes in 35 eyes and reported a VMT release rate of 43\% (15/35 eyes) and FTMH closure rate of $17 \%$ ( $1 / 6$ eyes). The authors reported the following factors to be associated with release of VMT: small adhesion diameter (mean 346 versus $730 \mu \mathrm{m}, p=0.05$ ), transient outer layer abnormalities on OCT $(p=0.008)$, younger age $(p=0.04)$, shorter duration of VMT (mean 4.6 versus 10.4 months, $p=0.03)$, and absence of concurrent retinal disease $(p=0.02)$. Sharma et al. published outcomes of 58 eyes treated for VMT and FTMH. At mean follow-up of 8.7 months, overall VMT release was observed

Table 2 Reported factors associated with successful pharmacologic vitreomacular traction (VMT) release with ocriplasmin $(0.125 \mathrm{mg})$

\section{Phakic status}

Age younger than 65 years

Small adhesion diameter $(\leq 1500 \mu \mathrm{m})$

Presence of full thickness macular hole

Absence of epiretinal membrane

Absence of concurrent retinal disease

Shorter duration of VMT

Transient outer retinal layer abnormalities on OCT

Macular hole size $<250 \mu \mathrm{m}$

Macular hole 'width factor ${ }^{18}$ (basal diameter-minimum linear diameter) $<60 \mu \mathrm{m}$ 
in 29/58 eyes (50\%) including in 4/15 eyes (27\%) with FTMH. While not achieving statistical significance, the authors also noted phakic status, age <65 years, absence of epiretinal membrane to be associated with VMT release, and greater FTMH closure in eyes with hole sizes $<400 \mu \mathrm{m}$.

Given that factors associated with VMT release were reported by various authors, Chatziralli et al. performed a meta-analysis to determine pooled odds ratios and confidence intervals (CI) for each factor reported to be associated with VMT release in the literature [15]. The following results were noted: age $<65$ years (OR 2.69, 95\% CI 1.79-4.03), female gender (OR 2.37, 95\% CI 1.23-4.57), adhesion diameter $<1500 \mu \mathrm{m} \quad(\mathrm{OR} \quad 7.85, \quad 95 \% \quad \mathrm{CI}$ 3.80-16.24), phakic lens status (OR 3.02, 95\% CI 2.02-4.50), absence of ERM (OR 4.75, 95\% CI 3.06-7.37), and FTMH size $<250 \mu \mathrm{m}$ (OR 2.25, 95\% CI 1.12-4.53).

\section{Macular Hole Closure}

In the MIVI-TRUST trials, overall nonsurgical macular hole closure rate was noted to be $40.6 \%$ with ocriplasmin treatment, with success noted for both small $(58.3 \%$ versus $16.0 \%, p<0.001)$ and medium $(36.8 \%$ versus $5.3 \%, p=0.009)$ holes. However, "real world" outcomes of hole closure have been lower than that found in the MIVI-TRUST trials, including in series with small and medium sized FTMH. For instance, Miller et al. [16] and Sharma et al. [14] reported a hole closure rates of $12.5 \%$ ( $1 / 8$ eyes) and $28.6 \%$ (4/14 eyes), respectively, despite that macular holes were sized $<400 \mu \mathrm{m}$.

Further subgroup analysis of macular hole cases has revealed additional insights. For instance, Dugel et al. [17] noted that while absence of epiretinal membrane was associated with overall VMT release, rates of macular hole closure were found to be similar in eyes with or without concurrent epiretinal membrane (38.9\% versus $41.5 \%$, respectively) in the MIVI-TRUST trials. In addition, Dugel et al. also noted that of eyes treated with ocriplasmin who exhibited hole closure at 6 months (43 eyes), 55.8\% exhibited VMA release at day 28 versus $44.2 \%$ who did not. Thus, a clear association between presence or absence of epiretinal membrane and VMA release with hole closure was not present in the MIVI-TRUST trial data.

To better identify OCT features that may predict hole closure other than hole size, Steel et al. prospectively evaluated outcomes in 31 eyes with idiopathic small or medium FTMH treated with ocriplasmin [18]. Mean hole size was $278 \mu \mathrm{m}$ (range $80-395 \mu \mathrm{m}$ ). In addition to hole size (defined by the minimal linear diameter), the authors evaluated the association between macular hole basal diameter and a new index termed the 'width factor' (defined as basal diameter minus the minimal linear diameter). Overall, the authors noted a VMA release rate of $61 \%$ (19/31 eyes) and a hole closure rate of $35 \%$ (11/31 eyes). All patients with hole closure exhibited VMA release. On multivariate analysis, the authors noted that 'width factor' was most strongly predictive of hole closure $(p<0.001)$, with a 'width factor' of $<60 \mu \mathrm{m}$ associated with hole closure in $95 \%$ of cases. Notably, in these series of small and medium FTMH, hole size alone was not significantly predictive of VMA release or hole closure.

Lastly, macular hole reopening has been reported in the literature following prior hole closure with ocriplasmin [19]. This was also noted in the MIVI-TRUST trial, observed in 4/43 eyes $(9.3 \%)$ treated with ocriplasmin at 6 months [6]. Prior authors have posited tractional forces from epiretinal membrane 
formation as a possible etiology of hole reopening post-ocriplasmin treatment [19].

Further data from prospective trials would be helpful in clarifying factors associated with macular hole closure, including the role of VMA release and concurrent epiretinal membrane, as a clear association between these factors and macular hole closure is not currently present. Such data may further help refine ideal macular hole candidates, and may explain why lower hole closure rates have been reported in the literature compared to MIVI-TRUST data.

\section{OCULAR ADVERSE EVENTS}

Ocular adverse events after use of this first-in-class pharmacologic agent have received special attention. In the phase III MIVI-TRUST trials, patients receiving ocriplasmin were more likely to experience transient blurred vision $(8.6 \%$ vs. $3.2 \%$, $p=0.01)$ and visual impairment $(5.4 \%$ vs. $1.6 \%, p=0.02$ ) compared to vehicle injection [6]. Following the commercial introduction of ocriplasmin, several case reports describing visual adverse events and abnormal OCT and/ or electroretinogram (ERG) findings were reported [20-23]. Authors have suggested the protease activity of ocriplasmin on laminin and other proteins present in the intraretinal space, and/or the physical effect of vitreous traction release, as a possible etiologic factor [24]; however, a precise mechanism for these adverse events and why such changes occur in only selected patients is currently uncertain.

\section{Categories of Adverse Events}

The American Society of Retinal Specialists (ASRS) Therapeutic Surveillance Committee
(TSC) evaluated both pre-marketing (clinical trial data) and post-marketing adverse event reports to better characterize ocular adverse events presumed to be related to ocriplasmin injection [25]. The following categories of related adverse events were identified: acute reduction in visual acuity, ERG changes, dyschromatopsia, retinal tear or detachment, lens subluxation or phacodonesis, abnormal pupillary reflex, retinal vascular changes, and OCT ellipsoid zone alterations. Shah et al., via an online distributed survey to vitreoretinal specialists, also sought to better characterize the nature and rate of adverse events following ocriplasmin injection, receiving a response rate of $11 \%$ (270/2465 respondents) [26].

Considering data from the MIVI-TRUST trials (Phase III data), the TSC analysis (voluntary post-marketing event reports), and results from the survey by Shah et al. (voluntary survey), the following incidence of related or possibly related adverse events in the literature include the following: acute reduction in visual acuity (1.3-16.95\%); dyschromatopsia (0.5-9.09\%); retinal tear or detachment (0.4-2.65\%); lens subluxation or phacodonesis (0.02-0.38\%); abnormal pupillary reflex (0.3-1.8\%); and retinal vascular changes $(0.05-0.28 \%)[6,25,26]$.

Data from the OASIS study did not reveal any additional safety concerns or events with follow-up to 24 months, and provided time to resolution for many common visual complaints post ocriplasmin injection. Notably, 27/146 patients $(18.5 \%)$ in the ocriplasmin group reported 'visual impairment' and an additional $13 / 146$ patients $(8.9 \%)$ reported 'blurred vision' in the OASIS trial [10]. Symptoms resolved in 39 of these 41 eyes (95\%), with a median time to resolution of 47 days in patients reporting blurred vision and 16 days in those who reported visual impairment. 
The TSC acknowledged that analysis of post-marketing adverse events reports is insufficient to determine a true incidence of adverse events, as the voluntary and nonspecific nature of the data gathered results in both under-reporting and incomplete or non-standardized follow-up reporting [25]. Caution should be applied to evaluation of this information, as it may be subject to over-interpretation, under-reporting, selection bias, and recall bias.

\section{Optical Coherence Tomography Changes Post-Ocriplasmin Use}

Several post-marketing reports have described the nature of OCT changes following ocriplasmin use, including ellipsoid layer disruption and accumulation of subretinal fluid (SRF, Fig. 2) [14, 27-30]. Notably, ellipsoid zone alterations were not reported in the MIVI-TRUST trial data, likely owing to the use of time-domain OCT rather than spectral-domain OCT (SD-OCT).

Larger case series have similarly observed transient ellipsoid layer abnormalities on OCT in $29-56 \%$ of treated patients $[11,13,27,28]$, with OCT changes more common in patients with VMT release [13, 28]. For instance, Quezada-Ruiz et al. similarly reported outcomes in 23 eyes receiving ocriplasmin for VMT or FTMH and detailed the presence or absence of ellipsoid layer changes [28]. Overall, VMT release was noted in $11 / 23$ eyes $(47.8 \%)$ and FTMH closure noted in $2 / 8$ eyes (25\%). Overall, 10/23 eyes (43.47\%) exhibited ellipsoid layer alterations on OCT, of which seven eyes (70\%) exhibited VMT release and four eyes (40\%) exhibited acute visual acuity loss of two lines or more. Ellipsoid layer alterations were resolved in all eyes at one-month post injection.

Additional studies have sought to further quantify and characterize ellipsoid changes as

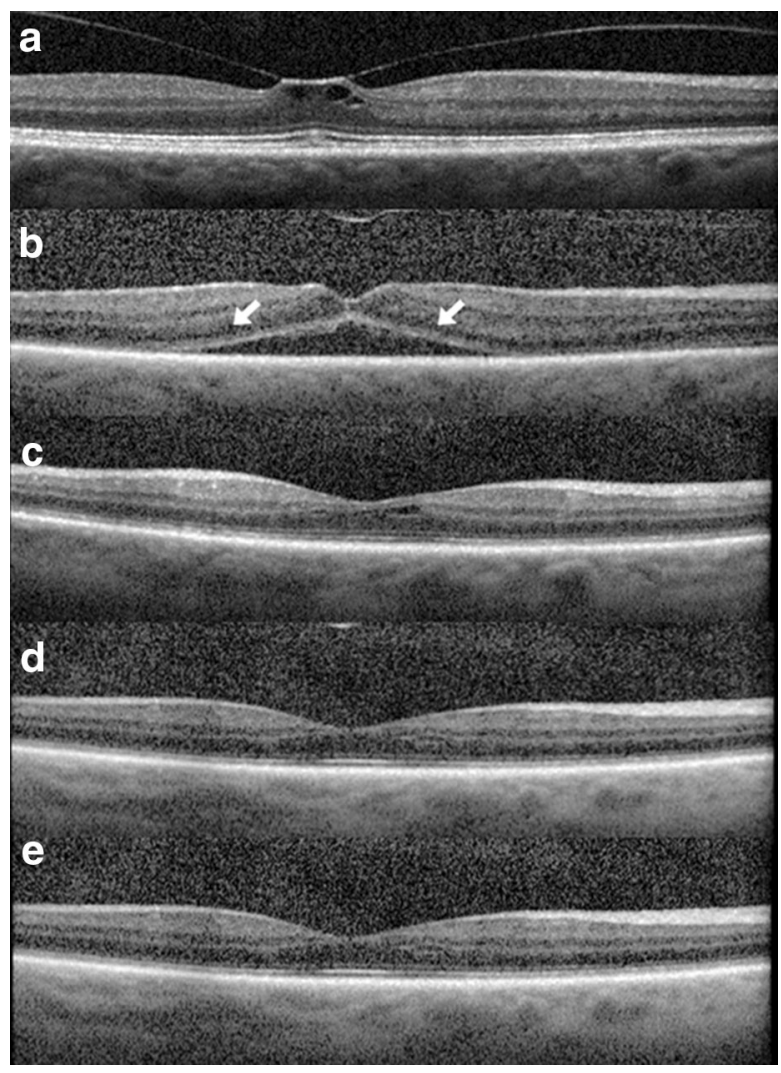

Fig. 2 Optical coherence tomography alterations post ocriplasmin use. A 62-year-old male with VMT of the right eye (a). Following intravitreal injection of ocriplasmin, VMT was released (b) at day 7. Ellipsoid layer attenuation and accumulation of subretinal fluid was present (arrows) and vision decreased from 20/50 at baseline to 20/80. Ellipsoid layer changes and subretinal fluid progressively resolved as shown at day $30(\mathbf{c})$, day 60 (d), and day 90 (e). Visual acuity was $20 / 25$ on day 60 and was maintained through the follow-up period thereafter

well as presence of SRF. For instance, Itoh and Ehlers quantitatively analyzed SD-OCT changes in 19 eyes receiving ocriplasmin for VMT [30]. In this series, VMT release was noted in $9 / 19$ eyes (47\%). Overall, 10/19 eyes (53\%) exhibited transient ellipsoid layer disruption with mean outer retinal thickness significantly reduced at 1 week post ocriplasmin injection $(p<0.01)$ with resolution at 1 month $(p=0.09)$ and 3 months $(p=0.91)$ versus baseline values. In addition, the authors noted a correlation between SRF accumulation and reduction in 
ellipsoid zone-retinal pigment epithelium (EZ-RPE) height $(p=0.00021$, correlation coefficient 0.88). Nudleman et al. similarly evaluated ellipsoid layer alterations and SRF accumulation in a series of 36 eyes with VMT and/or small to medium sized FTMH [27]. VMT release was noted in 15/36 eyes (42\%), and macular hole closure was observed in $7 / 9$ eyes (78\%). Of the eyes with VMT release, $73 \%$ of eyes (11/15 eyes) exhibited SRF accumulation compared to $19 \%$ of eyes (4/21 eyes) without VMT release. Overall, ellipsoid layer alterations were noted in $56 \%$ of eyes (20/36 eyes), with resolution in all cases at 1 year.

\section{Electroretinogram Changes \\ Post-Ocriplasmin Use}

A total of 18 reports of ERG changes were noted in the MIVI-TRUST data, TSC analysis, and survey of Shah et al. combined [6, 25, 26]. Two case reports with full-field ERG findings are also available in the literature, one with and one without FTMH. [21, 22] Findings were notable for reduction in $\mathrm{B}$ wave amplitudes during the scotopic ERG. Although A wave and photopic abnormalities were also present, the authors reported ERG findings were thought to be most consistent with bipolar cell dysfunction and reduced activity in primarily rod photoreceptors.

The ERG sub-study of the OASIS trial was completed to further clarify and describe ERG changes over the 24-month follow-up period [10]. Of the 220 patients enrolled in OASIS, 61 patients were ultimately analyzed in the ERG sub-study. Of these 61 patients, 40 patients were randomized to receive ocriplasmin and 21 to receive sham injection. Patients underwent full-field ERG following pupil dilation and dark adaptation. A reportable ERG change was defined in the sub-study as a $>40 \%$ change from baseline as read by a masked ERG expert at central reading center [10].

In the sub-study, $16 / 40$ eyes (40\%) in the ocriplasmin group had study-defined observable ERG changes as compared to $1 / 21$ eyes (4.8\%) in the sham group [10]. The data reported indicated that ERG changes were more likely to occur in eyes with VMT release and, over the course of the study, eyes with ERG changes maintained or gained visual acuity. Of the 16 eyes with ERG changes in the ocriplasmin group, 13 eyes (81.3\%) had resolution of ERG changes by the conclusion of the study with a median time to resolution of 6 months (range 21-449 days). Notably, a majority of eyes (10/16 eyes, $62.5 \%$ ) with ERG changes in the ocriplasmin group exhibited VMT release by day 28 , and a greater percentage (15/16 eyes, $94 \%)$ maintained or gained visual acuity at study end. The ERG results, however, must be interpreted in light of study limitations, including that only $28 \%$ of patients in the OASIS trial (61/220 patients) were analyzed in the sub-study.

Long-term ramifications of OCT and ERG abnormalities are not fully known and continue to be investigated, but appear to be related to pharmacologic VMT release with ocriplasmin. The interesting lack of correlation with ultimate visual acuity outcomes is also interesting and worth noting. To date, all cases with reported long-term outcomes have been transient with restoration of visual acuity [10, 20, 22, 23, 27].

\section{VITRECTOMY OUTCOMES FOLLOWING OCRIPLASMIN USE}

Vitrectomy has been shown to be an effective treatment option for patients with VMT, with well-characterized rare adverse events including infection, hemorrhage, retinal detachment, 
macular hole, and loss of vision $[4,5]$. In the MIVI-TRUST trials, a total of 57/341 ocriplasmin-treated eyes (17.7\%) and 46/169 placebo eyes (27.2\%) that did not achieve primary study endpoints by day 28 ultimately underwent vitrectomy for VMT treatment by month 6. In addition, of eyes that did meet primary endpoints, 25/123 ocriplasmin treated eyes (20.3\%) and 4/19 placebo eyes (21.1\%) underwent vitrectomy by month 6 [6].

Surgical outcomes, intraoperative complications, and postoperative complications in patients undergoing vitrectomy for VMT following use of ocriplasmin have been favorable (Fig. 3). In a multicenter, controlled interventional series, Greven et al. retrospectively evaluated visual acuity and surgical outcomes in 51 eyes of 51 patients undergoing vitrectomy for VMT or FTMH who had already been treated with ocriplasmin. Outcomes were compared to a control group comprised of 22 eyes of 22 patients who had been offered ocriplasmin but chose vitrectomy alone. Anatomic success (release of VMT or closure of FTMH) was achieved in 98\% (50/51 eyes) of eyes in the ocriplasmin treated group and 100\% (22/22 eyes) in the control group. At 6 months, the mean change in vision was similar between the ocriplasmin and control groups $(-0.17$ versus -0.23 LogMAR, $p=0.57$ ).

Other authors have noted that OCT findings commonly observed after ocriplasmin use, including ellipsoid layer changes and SRF accumulation, may continue to be observed following subsequent vitrectomy. For instance, Hager et al. reported a series of four eyes undergoing vitrectomy for persistent VMT after ocriplasmin. The authors noted the presence of SRF in 3/4 eyes (75\%), with SRF resolution observed in all cases at 7 months postoperatively.

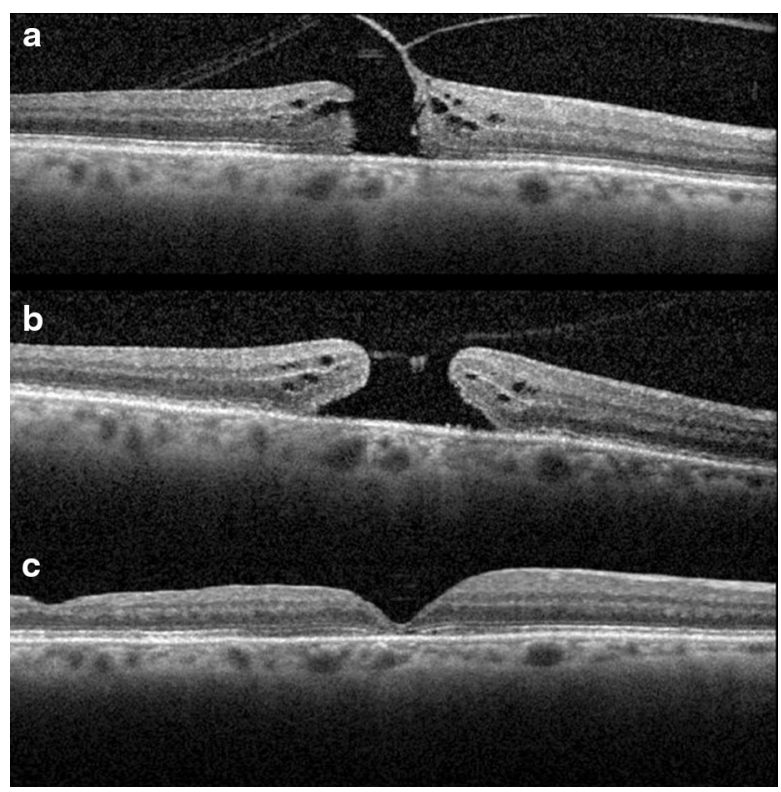

Fig. 3 Surgical closure of macular hole after prior treatment with ocriplasmin. A 6-year-old phakic male with medium sized FTMH (a). Visual acuity was 20/100 at baseline. Following intravitreal injection of ocriplasmin, the macular hole enlarged and VMT did not release (b). Vision worsened to 20/400. Following vitrectomy surgery, macular hole closure was achieved and vision improved to 20/80 (c) as shown on post-operative day 60

\section{CONCLUSION}

Data from Phase III Clinical Trials and post-marketing studies have expanded our understanding of pharmacologic vitreolysis with ocriplasmin. Ocriplasmin has been demonstrated as an effective nonsurgical therapy for VMT release and FTMH closure, with patients on average experiencing improvement in best-corrected visual acuity and visual function. Importantly, careful patient selection improves VMT release success rates. Ocriplasmin has been carefully monitored for adverse events, and these, particularly acute but almost entirely self-limited visual disturbances, are important for patient consent and understanding. Structural changes on OCT are common and often related to successful VMT release, the etiology of which remains 
incompletely understood. Overall, adverse events have been largely transient, with restoration of visual acuity; however, in select patients, alterations may persist. Surgical outcomes in eyes with a prior history of ocriplasmin treatment have been favorable and comparable to control eyes.

Future areas of interest include the efficacy of ocriplasmin for VMT in patients with concurrent macular disease and the role of ocriplasmin for other vitreoretinal surgery indications, including pediatric retinal detachment and diabetic tractional detachment. Additionally, trials directly comparing outcomes with pneumatic vitreolysis (intraocular perfluropropane gas or sulfur hexafluoride gas) would be of special interest in determining the relative efficacy of ocriplasmin compared to treatment alternatives. Prospective, phase IV studies in addition to improved post-marketing reporting of adverse events will be helpful in refining the ongoing risk/benefit profile of ocriplasmin use.

\section{ACKNOWLEDGMENTS}

No funding or sponsorship was received for this study or publication of this article. All named authors meet the International Committee of Medical Journal Editors (ICMJE) criteria for authorship for this manuscript, take responsibility for the integrity of the work as a whole, and have given final approval to the version to be published.

Disclosures. M Ali Khan has nothing to disclose. Julia A. Haller has been a consultant for ThromboGenics, Inc.

Compliance with ethics guidelines. This article is based on previously conducted studies, and does not involve any new studies of human or animal subjects performed by any of the authors.

Open Access. This article is distributed under the terms of the Creative Commons Attribution-NonCommercial 4.0 International License (http://creativecommons.org/licenses/ by-nc/4.0/), which permits any noncommercial use, distribution, and reproduction in any medium, provided you give appropriate credit to the original author(s) and the source, provide a link to the Creative Commons license, and indicate if changes were made.

\section{REFERENCES}

1. Sebag J. Age-related changes in human vitreous structure. Graefes Arch Clin Exp Ophthalmol. 1987;225(2):89-93.

2. Duker JS, Kaiser PK, Binder S, de Smet MD, Gaudric A, Reichel E, et al. The International Vitreomacular Traction Study Group classification of vitreomacular adhesion, traction, and macular hole. Ophthalmology. 2013;120(12):2611-9.

3. Gass J. Stereoscopic atlas of macular diseases: diagnosis and treatment. 4th ed. St. Louis: Mosby; 1997.

4. Jackson TL, Nicod E, Angelis A, Grimaccia F, Prevost AT, Simpson ARH, et al. Pars plana vitrectomy for vitreomacular traction syndrome: a systematic review and metaanalysis of safety and efficacy. Retina. 2013;33(10):2012-7.

5. Chang JS, Smiddy WE. Cost evaluation of surgical and pharmaceutical options in treatment for vitreomacular adhesions and macular holes. Ophthalmology. 2014;121(9):1720-6.

6. Stalmans P, Benz MS, Gandorfer A, Kampik A, Girach A, Pakola S, et al. Enzymatic vitreolysis with ocriplasmin for vitreomacular traction and macular holes. N Engl J Med. 2012;367(7):606-15.

7. Varma R, Haller JA, Kaiser PK. Improvement in patient-reported visual function after ocriplasmin for vitreomacular adhesion: results of the Microplasmin for Intravitreous Injection-Traction Release Without Surgical Treatment 
(MIVI-TRUST) Trials. JAMA Ophthalmol. 2015;133(9):997-1004.

8. Gandorfer A, Benz MS, Haller JA, Stalmans P, Pakola SJ, Girach A, et al. Association between anatomical resolution and functional outcomes in the mivi-trust studies using ocriplasmin to treat symptomatic vitreomacular adhesion/ vitreomacular traction, including when associated with macular hole. Retina. 2015;35(6):1151-7.

9. Haller JA, Stalmans P, Benz MS, et al. Efficacy of intravitreal ocriplasmin for treatment of vitreomacular adhesion: subgroup analyses from two randomized trials. Ophthalmology. 2015;122(1):117-22.

10. Kaiser PK. The OASIS study: evaluating ocriplasmin for the treatment of symptomatic VMA/VMT including macular hole over 2 years. In: American Academy of Ophthalmology annual meeting retina subspecialty day, Las Vegas, NV; 2015.

11. Singh RP, Li A, Bedi R, Srivastava S, Sears JE, Ehlers $\mathrm{JP}$, et al. Anatomical and visual outcomes following ocriplasmin treatment for symptomatic vitreomacular traction syndrome. $\mathrm{Br} \mathrm{J}$ Ophthalmol. 2014;98(3):356-60.

12. Kim BT, Schwartz SG, Smiddy WE, Doshi RR, Kovach JL, Berrocal AM, et al. Initial outcomes following intravitreal ocriplasmin for treatment of symptomatic vitreomacular adhesion. Ophthalmic Surg Lasers Imaging Retina. 2013;44(4):334-43.

13. Warrow DJ, Lai MM, Patel A, Raevis J, Berinstein DM. Treatment outcomes and spectral-domain optical coherence tomography findings of eyes with symptomatic vitreomacular adhesion treated with intravitreal ocriplasmin. Am J Ophthalmol. 2015;159(1):20.e1-30.e1.

14. Sharma P, Juhn A, Houston SK, Fineman M, Chiang A, Ho A, et al. Efficacy of intravitreal ocriplasmin on vitreomacular traction and full-thickness macular holes. Am J Ophthalmol. 2015;159(5):861.e2-867.e2.

15. Chatziralli I, Theodossiadis G, Xanthopoulou P, Miligkos M, Sivaprasad S, Theodossiadis P. Ocriplasmin use for vitreomacular traction and macular hole: a meta-analysis and comprehensive review on predictive factors for vitreous release and potential complications. Graefes Arch Clin Exp Ophthalmol. 2016;254(7):1247-56.

16. Miller JB, Kim LA, Wu DM, Vavvas DG, Eliott D, Husain D. Ocriplasmin for treatment of stage 2 macular holes: early clinical results. Ophthalmic Surg Lasers Imaging Retina. 2014;45(4):293-7.
17. Dugel PU, Regillo C, Eliott D. characterization of anatomic and visual function outcomes in patients with full-thickness macular hole in ocriplasmin phase 3 trials. Am J Ophthalmol. 2015;160(1):94.e1-99.e1.

18. Steel DHW, Parkes C, Papastavrou VT, Avery PJ, El-Ghrably IA, Habib MS, et al. Predicting macular hole closure with ocriplasmin based on spectral domain optical coherence tomography. Eye. 2016;30(5):740-5.

19. Khan MA, Shahlaee A, Ho AC. Reopening of a full-thickness macular hole 2 years after pharmacologic closure with ocriplasmin. JAMA Ophthalmol. 2015;17:1-2.

20. Freund KB, Shah SA, Shah VP. Correlation of transient vision loss with outer retinal disruption following intravitreal ocriplasmin. Eye. 2013;27(6):773-4.

21. Fahim AT, Khan NW, Johnson MW. Acute panretinal structural and functional abnormalities after intravitreous ocriplasmin injection. JAMA Ophthalmol. 2014;132(4):484-6.

22. Tibbetts MD, Reichel E, Witkin AJ. Vision loss after intravitreal ocriplasmin: correlation of spectral-domain optical coherence tomography and electroretinography. JAMA Ophthalmol. 2014;132(4):487-90.

23. Quezada Ruiz C, Pieramici DJ, Nasir M, Rabena M, Avery RL. Severe acute vision loss, dyschromatopsia, and changes in the ellipsoid zone on SD-OCT associated with intravitreal ocriplasmin injection. Retin Cases Brief Rep. 2015;9(2):145-8.

24. Beebe DC. Understanding the adverse effects of ocriplasmin. JAMA Ophthalmol. 2015;133(2):229.

25. Hahn P, Chung MM, Flynn HW, Huang SS, Kim JE, Mahmoud TH, et al. Safety profile of ocriplasmin for symptomatic vitreomacular adhesion: a comprehensive analysis of premarketing and postmarketing experiences. Retina. 2015;35(6):1128-34.

26. Shah SP, Jeng-Miller KW, Fine HF, Wheatley HM, Roth DB, Prenner JL. Post-marketing survey of adverse events following ocriplasmin. Ophthalmic Surg Lasers Imaging Retina. 2016;47(2):156-60.

27. Nudleman E, Franklin MS, Wolfe JD, Williams GA, Ruby AJ. Resolution of subretinal fluid and outer retinal changes in patients treated with ocriplasmin. Retina. 2016;36(4):738-43.

28. Quezada-Ruiz C, Pieramici DJ, Nasir M, Rabena M, Steinle N, Castellarin AA, et al. Outer retina 
reflectivity changes on SD-OCT after intravitreal ocriplasmin for vitreomacular traction and macular hole. Retina. 2015;35(6):1144-50.

29. Itoh Y, Kaiser PK, Singh RP, Srivastava SK, Ehlers JP. Assessment of retinal alterations after intravitreal ocriplasmin spectral-domain optical coherence tomography. Ophthalmology. 2014;121(12):2506.e2-2507.e2.

30. Itoh Y, Ehlers JP. Ellipsoid zone mapping and outer retinal characterization after intravitreal ocriplasmin. Retina. 2016. doi:10.1097/IAE. 0000000000001110 